\title{
Was ist gesichert in der Behandlung der diabetischen Nephropathie?
}

\author{
S. M. Kohler und B. K. Krämer \\ Klinik und Poliklinik für Innere Medizin II (Direktor: Prof. Dr. A. J. G. Riegger), Universitätsklinikum Regensburg
}

Bei einem 10-20 Jahre bestehenden Diabetes mellitus Typ I entwickelt sich in etwa $40 \%$ eine diabetische Nephropathie mit Proteinurie, arterieller Hypertonie und Abnahme der glomerulären Filtrationsrate, die nach weiteren 5 Jahren in aller Regel in das Endstadium der dialysepflichtigen Niereninsuffizienz übergeht $(15,17,23)$ (Abbildung 1).

In verschiedenen tierexperimentellen Studien wurde durch ACE(Angiotensin-ConvertingEnzym)-Hemmer ein Anstieg des glomerulären Kapillardrucks und das Auftreten einer Albuminurie bei diabetischen Ratten verhindert $(1,36)$. Andere Autoren bezweifeln die günstige Wirkung von ACEHemmern auf diabetische Ratten (9). Durch Gabe von Insulin bei diabetischen Ratten wurde ebenfalls eine Abnahme des glomerulären Kapillardrucks erzielt (30).

Als frühes Vorhersagekriterium einer diabetischen Nephropathie gilt die Mikroalbuminurie mit einer Albuminausscheidung von 30-300 $\mathrm{mg} / 24 \mathrm{~h}(23,24,33)$.

Als Ursache der Mikroalbuminurie wird von einigen Autoren ein erhöhter glomerulärer Kapillardruck angesehen, von anderen die strukturelle Veränderung der Glomeruli und der glomerulären Basalmembran, die durch nicht-enzymatische Proteinglykosylierung und den Verlust anionischer Ladungen (mit der Bildung von »advanced glycosylation end products $\ll)$ entsteht $(17,23)$.

Im folgenden werden therapeutische Möglichkeiten bei Mikroalbuminurie und diabetischer Nephropathie (Albuminausscheidung $>300$ $\mathrm{mg} / 24 \mathrm{~h}$ ) erörtert, deren Wirksamkeit durch kontrollierte Langzeitstudien gesichert oder zumindest sehr wahrscheinlich gemacht wurde. Die große Mehrzahl dieser Studien betrafen den Typ-I-Diabetes.

Dtsch. med. Wschr. 118 (1993), 433-437

(c) Georg Thieme Verlag Stuttgart · New York

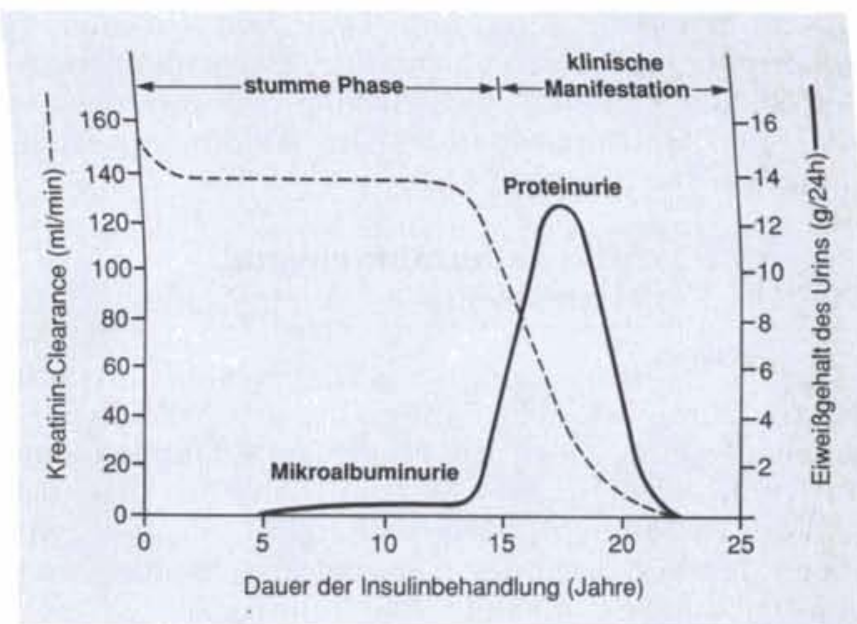

Abb. 1 Verlauf der diabetischen Nephropathie (nach Friedman und Beyer [12a]).

\section{Mikroalbuminurie und Normotonie}

Marre und Mitarbeiter (19) wiesen in einer placebo-kontrollierten Doppelblindstudie (20 Patienten, Beobachtungszeit 6 Monate) nach, daß die Therapie mit $20 \mathrm{mg} / \mathrm{d}$ Enalapril zu einer signifikanten Abnahme der Albuminausscheidung führte. Alle Patienten litten an einem insulinabhängigem Diabetes mellitus, wiesen vor Therapiebeginn eine Mikroalbuminurie und normale Blutdruckwerte auf.

Bei keinem der Patienten aus der Enalapril-Gruppe trat innerhalb eines Jahres eine diabetische Nephropathie auf (Placebo-Gruppe: $n=3$ ). Ferner kam es in der Therapiegruppe zu einer Abnahme des mittleren arteriellen Blutdrucks, so daß nicht zu klären war, ob die Abnahme der Mikroalbuminurie durch die Senkung des mittleren arteriellen Blutdrucks oder durch die dilatierende Wirkung des ACE-Hemmers auf das Vas efferens hervorgerufen wurde $(18,19)$. 
Von der »Melbourne Study Group « (21) konnte in der Beobachtungszeit von einem Jahr keine Abnahme der Albuminausscheidung festgestellt werden, weder mit Perindopril noch mit Nifedipin; aber auch keiner der Patienten erkrankte an einer diabetischen Nephropathie.

Cook und Mitarbeiter (8) stellten bei zwölf normotonen Kindern im Alter von ungefähr 14 Jahren während einer dreimonatigen Captopril-Therapie eine Abnahme der Albuminausscheidung fest. Mathiesen und Mitarbeiter (20) untersuchten 44 normotone Patienten mit Mikroalbuminurie und insulinabhängigem Diabetes mellitus über 4 Jahre und fanden in der Therapiegruppe $(25-100 \mathrm{mg} / \mathrm{d}$ Captopril, ab dem 30 . Monat kombiniert mit $2,5 \mathrm{mg} / \mathrm{d}$ Bendroflumethiazid) auch langfristig eine Abnahme der Albuminausscheidung und kein Fortschreiten zu einer diabetischen Nephropathie. Dagegen erkrankten sieben Patienten der Placebo-Gruppe an einer diabetischen Nephropathie. Die Albuminausscheidung lag über $300 \mathrm{mg} / 24 \mathrm{~h}$.

\section{Mikroalbuminurie und Hypertonie}

Im Verlauf eines Diabetes mellitus mit Mikroalbuminurie tritt häufig eine arterielle Hypertonie auf. Christensen und Mogensen (6) fanden eine direkte Korrelation zwischen der Dauer des Diabetes und der Zunahme der Mikroalbuminurie sowie zwischen der Zunahme der Albuminausscheidung und der Erhöhung des diastolischen Blutdrucks.

Bei zehn Patienten mit Diabetes mellitus Typ II, arterieller Hypertonie und Mikroalbuminurie wurde durch ein Saluretikum $(2,5 \mathrm{mg} / \mathrm{d}$ Indapamid) in einer Beobachtungszeit von 2 Jahren eine Abnahme der Mikroalbuminurie erreicht (13). Andere Autoren verglichen die Wirkung von ACEHemmern $(10-20 \mathrm{mg} / \mathrm{d}$ Enalapril oder $2-8 \mathrm{mg} / \mathrm{d}$ Perindopril) und Calciumantagonisten $(60-120 \mathrm{mg} / \mathrm{d}$ Nicardipin oder $20-80 \mathrm{mg} / \mathrm{d}$ Nifedipin) bei Patienten, die an Diabetes mellitus, Mikroalbuminurie und arterieller Hypertonie litten. Nach einer Beobachtungszeit von 4 Wochen bzw. 12 Monaten zeigten sich bezüglich Blutdrucksenkung, Abnahme der Mikroalbuminurie und der glomerulären Filtrationsrate keine signifikanten Unterschiede $(2,21)$.

\section{Nephropathie und Normotonie}

Bei 15 Patienten mit diabetischer Nephropathie und normalem Blutdruck bewirkte Captopril in einer Dosierung von $25-100 \mathrm{mg} / \mathrm{d}$ innerhalb eines Jahres einen Abfall des mittleren arteriellen Blutdrucks um $3 \mathrm{~mm} \mathrm{Hg}$, eine Abnahme der Albuminurie um $11 \%$ sowie eine Abnahme der glomerulären Filtrationsrate um $3 \mathrm{ml} / \mathrm{min} \cdot 1,73 \mathrm{~m}^{2}$ (26). In der Kontrollgruppe $(n=17)$ stieg der mittlere arterielle Blutdruck um $6 \mathrm{~mm} \mathrm{Hg}$, die Albuminurie nahm um $55 \%$ zu, die glomeruläre Filtrationsrate um $6 \mathrm{ml}$ min $1,73 \mathrm{~m}^{2} \mathrm{ab}$. Möglicherweise läßt sich durch ACE-Hemmer, die zu einer verstärkten Dilatation des Vas efferens führen und dadurch den glomerulären Kapillardruck vermindern, eine weitere Abnahme der glomerulären Filtrationsrate bei diabetischer Nephropathie verzögern oder verhindern, auch wenn noch keine systemische Hypertonie eingetreten ist.

\section{Nephropathie und Hypertonie}

Die diabetische Nephropathie geht in $50-70 \%$ der Fälle mit einer arteriellen Hypertonie einher, die zu einer weiteren Verschlechterung der Nierenfunktion führt (15). In einer ersten Langzeitstudie, in der eine kleine Zahl von Patienten mit Diabetes mellitus Typ I, Proteinurie und arterieller Hypertonie untersucht wurde, stellte Mogensen (22) fest, daß sich durch eine Therapie mit Propranolol, später mit Metoprolol $(100-200 \mathrm{mg} / \mathrm{d})$, die jährliche Abnahme der glomerulären Filtrationsrate und die Proteinurie vermindern ließen. Vier der sechs Patienten erhielten zur weiteren Blutdrucksenkung noch Furosemid $(40 \mathrm{mg} / \mathrm{d})$ oder $100-200 \mathrm{mg} / \mathrm{d}$ Hydralazin.

Auch in einer Langzeitstudie über 6 Jahre wurde durch gute Blutdruckeinstellung mit Metoprolol, Hydralazin und Furosemid die Abnahme der glomerulären Filtrationsrate und die Proteinurie bei elf Patienten mit Diabetes mellitus Typ I, Nephropathie und Hypertonie vermindert (25). Parving und Mitarbeiter (27) behandelten 18 Patienten, die an einer diabetischen Nephropathie und arterieller Hypertonie litten, mit dem ACE-Hemmer Captopril und dem Diuretikum Furosemid oder Bendroflumethiazid (Abbildung 2): In der Beobachtungszeit von $2 \frac{1}{2}$ Jahren nahm die Albuminausscheidung ab, die

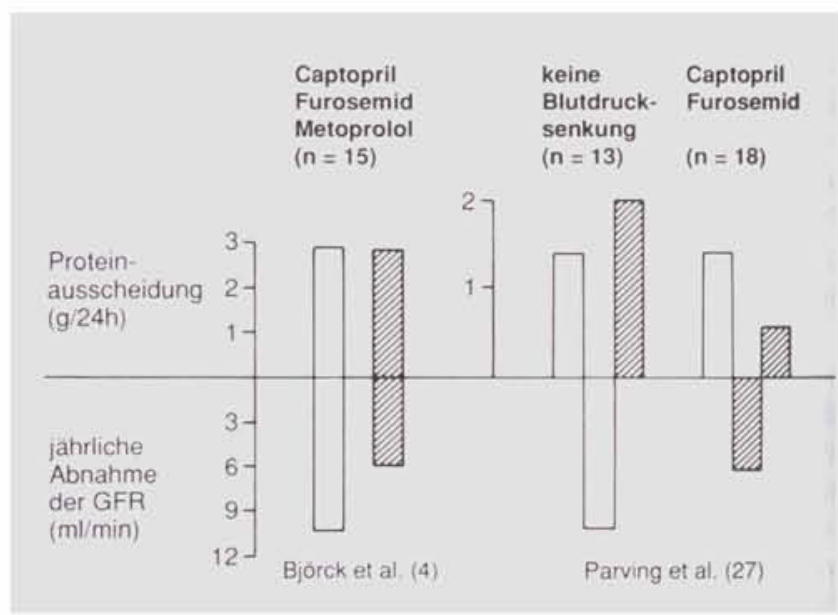

Abb. 2 Verminderung der Proteinurie und der Abnahme der glomerulären Filtrationsrate (GFR) durch Blutdrucksenkung bei diabetischer Nephropathie (nach Parving et al., Björk et al. $[4,27]$ ). Heller Balken = vor Therapie, dunkler Balken $=$ nach Therapie. 
Reduktion der glomerulären Filtrationsrate verlangsamte sich.

Die Senkung des mittleren arteriellen Drucks um $5 \mathrm{~mm} \mathrm{Hg}$ durch eine Kombinationstherapie mit ACE-Hemmer (Captopril), Diuretikum, $\beta$ Rezeptorenblocker und Calciumantagonisten verzögerte bei 15 Patienten mit diabetischer Nephropathie das Fortschreiten der Niereninsuffizienz (Abbildung 2) (4). In Mogensens Arbeitsgruppe wurde durch den Zusatz eines ACE-Hemmers zu der schon als wirksam erkannten blutdrucksenkenden Therapie eine weitere Abnahme der Proteinurie erreicht (28). Sie ging allerdings einher mit einer weiteren Senkung des mittleren arteriellen Drucks.

Die besonders günstige Wirkung von ACE-Hemmern auf Albuminausscheidung und glomeruläre Filtrationsrate bestätigte eine Studie, in der 22 Patienten mit Diabetes mellitus Typ I und diabetischer Nephropathie den ACE-Hemmer Enalapril $(5-20 \mathrm{mg} / \mathrm{d})$ erhielten, 18 Patienten den $\beta$-Rezeptorenblocker Metoprolol (maximal $200 \mathrm{mg} / \mathrm{d}$ ) (3). Beide Gruppen erhielten zusätzlich noch ein Diuretikum. In der Beobachtungszeit von 2 Jahren betrug die jährliche Abnahme der glomerulären Filtrationsrate $2,0 \mathrm{ml} / \mathrm{min}$ in der Enalapril-Gruppe, in der Metoprolol-Gruppe $5,6 \mathrm{ml} / \mathrm{min}$. Die Albuminausscheidung war in der Enalapril-Gruppe um $60 \%$ niedriger. Anzumerken ist jedoch, daß der diastolische Blutdruck im Liegen in der Enalapril-Gruppe um $6 \mathrm{~mm} \mathrm{Hg}$ niedriger war als in der Metoprolol-Gruppe, bei nahezu gleichem mittlerem arteriellem Blutdruck. Bei einem Patienten mußte die Enalapril-Therapie wegen ansteigender Kreatininwerte abgebrochen werden.

Die vorteilhafte Wirkung von ACEHemmern auf die glomeruläre Filtrationsrate konnte von Grönhagen-Riska und Mitarbeitern (14), die ebenfalls ACE-Hemmer und $\beta$-Rezeptorenblocker verglichen, nicht bestätigt werden. Diese Mitteilung liegt bisher jedoch nur als Abstract vor (14).

\section{Proteinrestriktion}

Ratten, bei denen durch Streptozocin ein Diabetes mellitus erzeugt wurde, wiesen nach Gabe eiweißarmen Futters eine verbesserte glomeruläre Hämodynamik auf. Ferner nahm die Proteinurie ab. Der histologische Schweregrad der diabetischen Nephropathie war nach 3 Monaten proteinarmer Kost deutlich geringer $(16,35)$.

In zwei Studien wurde bei 19 bzw. 20 Patienten mit diabetischer Nephropathie die Wirksamkeit einer Eiweißrestriktion auf $0,6-0,7 \mathrm{~g} / \mathrm{kg} \cdot \mathrm{d}$ untersucht $(34,37)$. Die Kontrollgruppen mit $19 \mathrm{bzw}$. 15 Patienten erhielten eine Eiweißzufuhrvon $1 \mathrm{~g} / \mathrm{kg} \cdot \mathrm{d}$. In der Beobachtungszeit von 37 bzw. 29 Monaten konnte eine Abnahme der Proteinurie und der Ver-

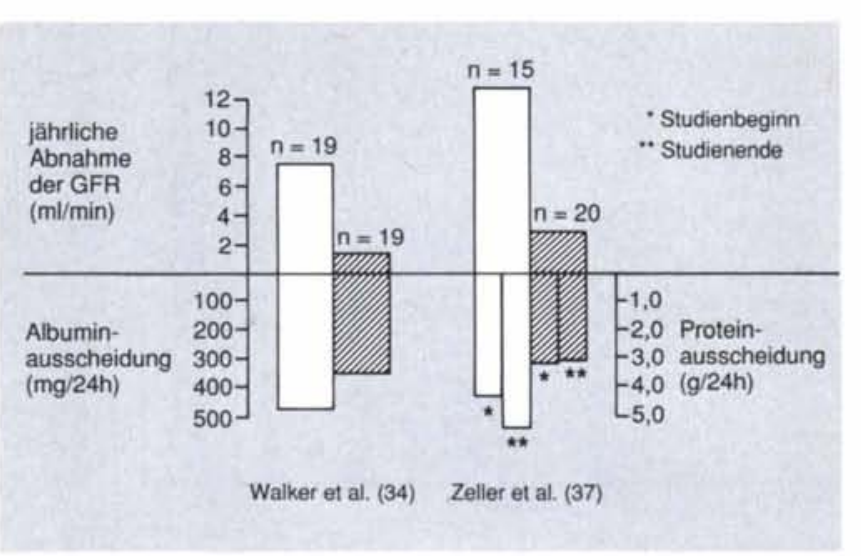

Abb. 3 Abnahme der Albuminausscheidung und Änderung der glomerulären Filtrationsrate (GFR) durch EiweiBrestriktion auf $0,6-0,7 \mathrm{~g} / \mathrm{kg} \cdot \mathrm{d}$ (nach Walker et al., Zeller et al. $[34,37])$. Heller Balken = Kontrollgruppe, dunkle Balken = Therapiegruppe.

ringerung der glomerulären Filtrationsrate in der Gruppe mit eiweißreduzierter Kost vermerkt werden (Abbildung 3). Zum gleichen Ergebnis kamen Brouhard und LaGrone (5): Durch Eiweißrestriktion wird das Fortschreiten der Niereninsuffizienz bei diabetischer Nephropathie verzögert, die Albuminurie nimmt ab.

In einer Crossover-Studie (Beobachtungszeit 3 Wochen) wurde die Eiweißaufnahme bei acht Patienten mit Diabetes mellitus Typ I, Mikroalbuminurie und normalem Blutdruck auf $38-57 \mathrm{~g} / \mathrm{d}$ beschränkt (7). Die Albuminausscheidung sank dadurch auf $22 \mathrm{mg} / \mathrm{d}$. Bei einer Eiweißzufuhr von $55-117 \mathrm{~g} / \mathrm{d}$ betrug sie $33 \mathrm{mg} / \mathrm{d}$. Diese Studie legt nahe, daß möglicherweise bereits im Stadium der Mikroalbuminurie durch Eiweißrestriktion eine Zunahme der Albuminausscheidung verhindert werden kann. Um dies zu belegen, sind aber noch Untersuchungen mit größeren Fallzahlen erforderlich.

\section{Normoglykämie und diabetische Nephropathie}

Unter der Vorstellung, durch eine optimale Langzeitblutzuckereinstellung, gemessen am $\mathrm{HbA}_{1 \mathrm{c}}$, die Entwicklung zur diabetischen Nephropathie zu verzögern oder zu verhindern, wurden in den letzten Jahren verschiedene Studien, zum Teil multizentrisch und über eine längere Beobachtungszeit, durchgeführt.

Tatsächlich ergab sich bei einem $\mathrm{HbA}_{1 \mathrm{c}}$-Wert von $8,1 \%$ in der Beobachtungszeit von 8 Monaten, in einer anderen Studie bei einem $\mathrm{HbA}_{1 \mathrm{c}^{-}}$ Wert von $7,2 \%$ über 2 Jahre eine Abnahme der Albuminausscheidung im 24-Stunden-Urin $(11,31$, 32) (Abbildung 4). Die Blutzuckereinstellung wurde mit einer Insulinpumpe erreicht. Ferner zeigten Reichard und Rosenquist (29), daß durch eine intensivierte konventionelle Insulinbehandlung ein $\mathrm{HbA}_{1 \mathrm{c}^{-}}$ 


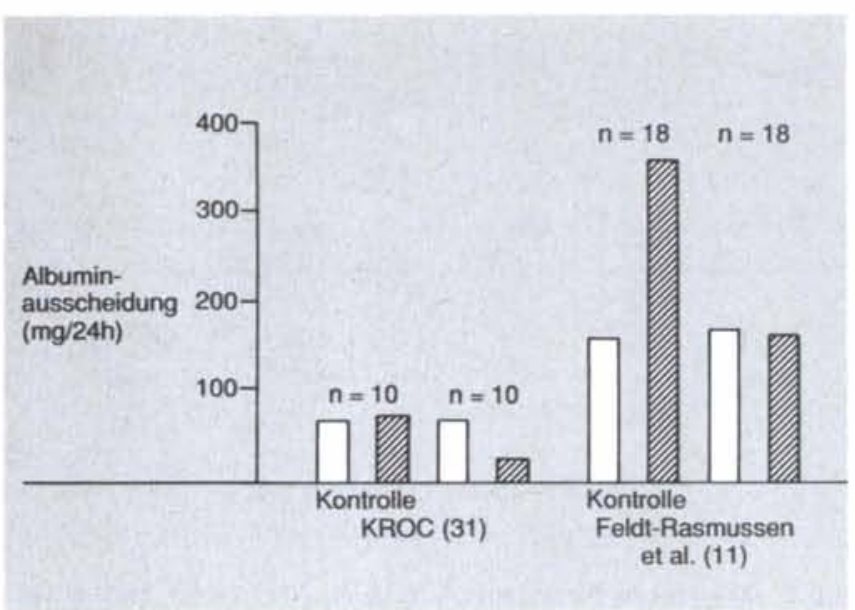

Abb. 4 Abnahme der Albuminausscheidung durch eine möglichst optimale Blutzuckereinstellung $\left(\mathrm{HbA}_{1 \mathrm{c}}\right.$-Werte unter $8 \%$ ) (nach [11, 31]). Heller Balken = vor Therapie, dunkler Balken = nach Therapie.

Wert von 7,4\% erreicht werden konnte, die konventionelle Therapie ergab Werte um 9\%. Die verbesserte Blutzuckereinstellung der 44 Patienten mit intensivierter Insulintherapie verzögerte - im Gegensatz zur konventionellen Therapie - das Fortschreiten der diabetischen Nephropathie. Die Beobachtungszeit betrug 3 Jahre.

In einer anderen Studie wurde die Blutzuckerkonzentration von 51 Patienten mit insulinabhängigem Diabetes mellitus und Mikroalbuminurie über 8 und 5 Jahre kontrolliert (12). Während der konventionellen Insulintherapie nahm die $\mathrm{Al}$ buminausscheidung weiter $\mathrm{zu}$, die glomeruläre Filtrationsrate ab. Bei intensivierter Insulintherapie blieben die Werte stabil: Nur einer von 32 Patienten mit geringer Mikroalbuminurie (65 mg/24h) erkrankte an einer diabetischen Nephropathie. Sie trat ferner bei zwei von neun Patienten auf, die eine Mikroalbuminurie von $100-300 \mathrm{mg} / 24 \mathrm{~h}$ aufwiesen. Von den zehn Patienten mit stärkerer Mikroalbuminurie (100-300 mg/24 h), die konventionell mit Insulin behandelt wurden, erkrankten alle an einer diabetischen Nephropathie.

Eine Abnahme der Mikroalbuminurie konnte in der Oslo-Studie (10) bei 45 Patienten mit insulinabhängigem Diabetes mellitus während einer Untersuchungszeit von 7 Jahren, bei $\mathrm{HBA}_{1 \mathrm{c}}$-Werten von $8,5 \%$, festgestellt werden, wohingegen $\mathrm{HBA}_{1 \mathrm{c}^{-}}$ Werte $>10 \%$ bei vier von 14 Patienten mit einer Zunahme der Albuminurie von mehr als $200 \mathrm{mg} / 24 \mathrm{~h}$ einhergingen (10). Die Blutzuckerkonzentration wurde bei zehn der 45 Patienten mit einer Insulinpumpe, bei 33 durch eine intensivierte Insulintherapie und bei zwei Patienten durch eine konventionelle Insulintherapie reguliert.

\section{Resümee}

Es gibt zunehmend Hinweise darauf, daß ACE-Hemmstoffe das Auftreten einer diabetischen Nephropathie bei Patienten verzögern, die bereits eine Mikroalbuminurie, aber noch normale Blutdruckwerte aufweisen. Besteht eine arterielle Hypertonie, so ist sowohl im Stadium der Mikroalbuminurie als auch bei offensichtlicher diabetischer Nephropathie die Senkung des Blutdrucks erforderlich, um eine Progression der Niereninsuffizienz zu verhindern. Die Überlegenheit einer bestimmten Gruppe von Antihypertonika (zum Beispiel ACEHemmer) ist hierfür unseres Erachtens bisher nicht ausreichend gesichert.

Auch eine Proteinrestriktion wirkt sich bei diabetischer Nephropathie günstig aus. Ob eiweißarme Kost im frühen Stadium der Krankheit vorteilhaft ist, kann noch nicht beantwortet werden, da ausführliche Studien bisher fehlen.

Eine gute Blutzuckereinstellung mit $\mathrm{HbA}_{1 \mathrm{c}}$-Werten unter $8 \%$ verzögert im Stadium der Mikroalbuminurie das Fortschreiten zur diabetischen Nephropathie. Ob sie die Progredienz einer bereits bestehenden diabetischen Nephropathie verhindert, ist noch unsicher.

Die genannten Therapieempfehlungen können bisher nur für Typ-I-Diabetiker als ausreichend gesichert gelten.

\section{Literatur}

1 Anderson, S., H. G. Rennke, D. L. Garcia, B. M. Brenner: Short and long term effects of antihypertensive therapy in the diabetic rat. Kidney int. 36 (1989), 526-536.

2 Baba, T., S. Murabayashi, K. Takebe: Comparison of the renal effects of angiotensin converting enzyme inhibitor and calcium antagonist in hypertensive type II (non-insulin-dependent) diabetic patients with microalbuminuria. A randomised controlled trial. Diabetologia 32 (1989), 40-44.

3 Björk, S., H. Mulec, S. A. Johnsen, G. Norden, M. Aurell: Renal protective effect of enalapril in diabetic nephropathy. Brit. med. J. 304 (1992), 339-343.

4 Björk, S., G. Nyberg, H. Mulec, G. Granerus, H. Herlitz, M. Aurell: Beneficial effects of angiotensin converting enzyme inhibition on renal function in patients with diabetic nephropathy. Brit. med. J. 293 (1986), 471-474.

5 Brouhard, B. H., L. LaGrone: Effect of dietary protein restriction on functional renal reserve in diabetic nephropathy. Amer. J. Med. 89 (1990), 427-431

6 Christensen, C. K., C. E. Mogensen: The course of incipient diabetic nephropathy. Studies of albumin excretion and blood pressure. Diabet. Med. 2 (1985), 97-102.

7 Cohen, D., R. Dodds, G. Viberti: Effect of protein restriction in insulin dependent diabetics at risk of nephropathy. Brit. med. J. 294 (1987), 795-798.

8 Cook, J., D. Daneman, M. Spino, E. Sochett, K. Perlman, J. W. Balfe: Angiotensin converting enzyme inhibitor therapy to decrease microalbuminuria in normotensive children with insulin-dependent diabetes mellitus. J. Pediat. 117 (1990), 39-45.

9 Cooper, M. E., J. R. Rumble, T. J. Allen, R. C. O’Brien, G. Jerums, A. E. Doyle: Antihypertensive therapy in a model 
combining spontaneous hypertension with diabetes. Kidney int. 41 (1992), 898-903.

10 Dahl-Jörgensen, K., T. Björo, P. Kierulf, L. Sandvik, H. J. Bangstad, K. F. Hanssen: Long-term glycemic control and kidney function in insulin-dependent diabetes mellitus. Kidney int. 41 (1992), 920-923.

11 Feldt-Rasmussen, B., E. R. Mathiesen, T. Deckert: Effect of two years of strict metabolic control on progression of incipient nephropathy in insulin-dependent diabetes. Lancet 1986/II, 1300-1304.

12 Feldt-Rasmussen, B., E. R. Mathiesen, T. Jensen, T. Lauritzen, T. Deckert: Effect of improved metabolic control on loss of kidney function in type I (insulin-dependent) diabetic patients. An update of the Steno studies. Diabetologia 34 (1991), 164-170.

12a Friedman, E. A., M. M. Beyer: Uremia in diabetics. The prognosis improves. Klin. Wschr. 58 (1980), 1023-1028.

13 Gambardella, S., S. Frontoni, M. G. Felici, V. Spallone, P. Gargiulo, S. Morano, G. Menzinger: Efficacy of antihypertensive treatment with indapamide in patients with noninsulindependent diabetes and persistent microalbuminuria. Amer. J. Cardiol. 65 (1990), 46-50.

14 Grönhagen-Riska, C., E. Honkanen, K. Metsärinne, K. Rosenlöf, 1. Tikkanen, F. Fyhrquist: ACE-inhibition versus conventional antihypertensive treatment ( $\beta$-blockade) in diabetic nephropathy. Amer. J. Hypertens. 3 (1990), 67 (Abstract).

15 Hasslacher, C., E. Ritz: Hypertonie und Diabetes mellitus. Internist 31 (1990), 180-190.

16 Hostetter, T. H., J. L. Olson, H. G. Rennke, M. A. Venkatachalam, B. M. Brenner: Hyperfiltration in remnant nephrons. A potentially adverse response to renal ablation. Amer. J. Physiol. 241 (1981), 85-93.

17 Kleophas, W., T. Voitz, H. Messner, F. A. Gries: Diabetische Nephropathie. Fortschr. Med. 108 (1990), 309-312.

18 Marre, M., G. Chatellier, H. Leblanc, T. T. Guyenne, J. Menard, P. Passa: Prevention of diabetic nephropathy with enalapril in normotensive diabetics with microalbuminuria. Brit. med. J. 297 (1988), 1092-1095.

19 Marre, M., H. Leblanc, L. Suarez, T. T. Guyenne, J. Menard, P. Passa: Converting enzyme inhibition and kidney function in normotensive diabetic patients with persistent microalbuminuria. Brit. med. J. 294 (1987), 1448-1452.

20 Mathiesen, E. R., E. Hommel, J. Giese, H.-H. Parving: Efficacy of captopril in postponing nephropathy in normotensive insulin dependent diabetic patients with microalbuminuria. Brit. med. J. 303 (1991), 81-87.

21 Melbourne Diabetic Nephropathy Study Group: Comparison between perindopril and nifedipine in hypertensive and normotensive diabetic patients with microalbuminuria. Brit. med. J. 302 (1991), 210-216.

22 Mogensen, C. E.: Long term antihypertensive treatment inhibiting progression of diabetic nephropathy. Brit. med. J. 285 (1982), 685-688.

23 Mogensen, C. E.: Microalbuminuria as a predictor of clinical diabetic nephropathy. Kidney int. 31 (1987), 673-689.

24 Mogensen, C. E.: Microalbuminuria predicts clinical proteinuria and early mortality in maturity onset diabetes. New Engl. J. Med. 310 (1984), 356-360.

25 Parving, H.-H., A. R. Andersen, U. M. Smidt, E. Hommel, E. R. Mathiesen, P. A. Svendson: Effect of antihypertensive treatment on kidney function in diabetic nephropathy. Brit. med. J. 294 (1987), 1443-1447.

26 Parving, H.-H., E. Hornmel, M. D. Nielsen, J. Giese: Effect of captopril on blood pressure and kidney function in normotensive insulin dependent diabetics with nephropathy. Brit. med. J. 299 (1989), 533-536.

27 Parving, H.-H., E. Hommel, U. M. Smidt: Protection of kidney function and decrease in albuminuria by captopril in insulin dependent diabetics with nephropathy. Brit. med. J. 297 (1988), 1086-1091.

28 Pedersen, M. M., K. W. Hansen, A. Schmitz, K. Sorensen, C. K. Christensen, C. E. Mogensen: Effects of ACE inhibition supplementary to beta blockers and diuretics in early diabetic nephropathy. Kidney int. 41 (1992), 883-890.
29 Reichard, P., U. Rosenquist: Nephropathy is delayed by intensified insulin treatment in patients with insulin-dependent diabetes mellitus and retinopathy. J. intern. Med. 226 (1989), 81-87.

30 Scholey, J. W., T. E. Meyer: Control of glomerular hypertension by insulin administration in diabetic rats. J. clin. Invest. 83 (1989), 1384-1389.

31 The KROC Collaborative Study Group: Blood glucose control and the evolution of diabetic retinopathy and albuminuria. New Engl. J. Med. 311 (1984), 365-372.

32 The KROC Collaborative Study Group: Collaborative studies of the effects of continuous subcutaneous insulin infusion in insulin-dependent diabetes mellitus. Diabetes 34, Suppl. 3 (1985). 87-89.

33 Viberti, G. C., R. J. Jarrett, U. Mahmud, R. D. Hill, A. Argyropoulos, H. Keen: Microalbuminuria as a predictor of clinical nephropathy in insulin-dependent diabetes mellitus. Lancet 1982/1, 1430-1432.

34 Walker, J. D., J. J. Bending, R. A. Dodds, M. B. Mattock, T. J. Murrells, H. Keen, G. C. Viberti: Restriction of dietary protein and progression of renal failure in diabetic nephropathy. Lancet 1989/II, 1411-1415.

35 Wen, S.-F., T.-P. Huang, A. V. Moorthy: Effects of low protein diet on experimental diabetic nephropathy in the rat. J. Lab. clin. Med. 106 (1985), 589-597.

36 Zatz, R., B. R. Dunn, T. W. Meyer, S. Anderson, H. G. Rennke, B. M. Brenner: Prevention of diabetic glomerulopathy by pharmacological amelioration of glomerular capillary hypertension. J. clin. Invest. 77 (1986), 1925-1930.

37 Zeller, K., E. Whittaker, L. Sullivan, P. Raskin, H. R. Jacobson Effect of restricting dietary protein on the progression of renal failure in patients with insulin-dependent diabetes mellitus. New Engl. J. Med. 324 (1991),78-84.

Dr. S. M. Kohler, Privatdozent Dr. B. K. Krämer

Klinik und Poliklinik für Innere Medizin II

der Universität

Franz-Josef-Strauß-Allee 11

W-8400 Regensburg 